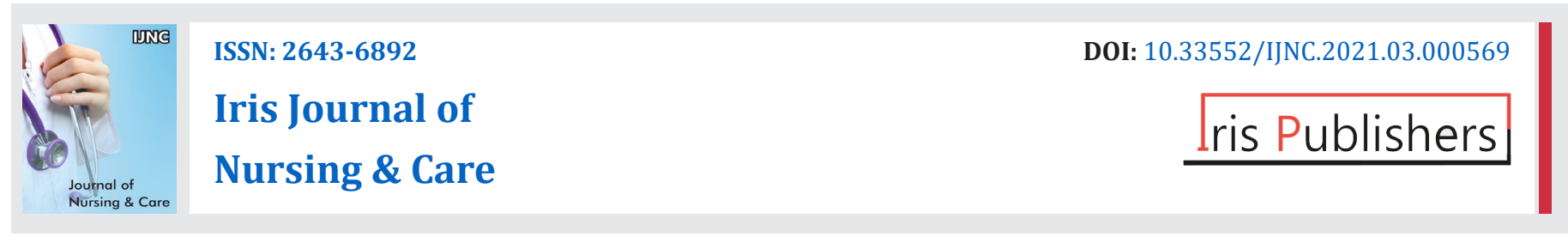

Short Communication

Copyright (C) All rights are reserved by Wen Hsing Yang

\title{
SARS, COVID-19 and Changing in Nursing Practice
}

\author{
Wen Hsing Yang* \\ Department of Nursing, Long Island University, USA
}

*Corresponding author: Wen Hsing Yang, Department of Nursing, Long Island University, New York, USA.

Received Date: February 23, 2021

Published Date: February 27, 2021

\section{Introduction}

SARS was a serious outbreak worldwide, resulting in studies in infection protocol and delivery of nursing care. This study compared conventional nursing practice with a modular design of nursing delivery by measuring the nature and frequency of nursing activities, efficiency, infection control practice, namely hand hygiene, as well as satisfaction levels among nurses and patients [1]. A descriptive and quasi-experimental study with modular nursing as the intervention, it consisted of a pre-intervention (T0) and two post-intervention phases (T1 and T2) in one medical and two surgical wards [1]. Several focus group meetings were conducted with senior nursing staffs from the three wards to enable the research team to acquaint staff with the model design and to solicit their input regarding proposed structure changes [1]. A series of educational sessions was provided to the staff. The data collection tools used to evaluate the modular nursing practice included: a work sampling, observation, checklist, focus interview with nurses, questionnaires to gauge nurses' perceived competency and caring attributes, patient satisfaction questionnaires and a hand hygiene audit. The data was collected and analyzed from observations and responses to the questionnaires. No significant changes in direct care were reported but there was an increase in patient/family education due to continuity of care [1]. Also, results showed a general increase in nurses' hand washing and personal break activities. However, due to time constraints on the nurses, a lapse on handwashing also was noted [1]. Increasing time in caring for patients showed a positive result in preventing urinary tract infection and reducing infection control. Handwashing was especially important in infection control. A stable nurse-patient ratio needs to be addressed to facilitate infection control.

\section{Nursing Practice}

COVID-19, a worldwide epidemic worse than SARS, is having a profound effect on nursing practice everywhere. First, the virus is new, the data on the virus was slowly realized and politically controlled by the original country, and the phenomenal rate of transmission was unexpected and deadly. The entire worldwide medical community was not prepared for this epidemic, even though some countries did have epidemic watchdog task forces. As the infection progressed and mortality increased, personal protective equipment (PPE) has shown to be the most necessary piece of equipment to protect medical staff from becoming infected by their patients [2]. Because of the rapid transmission through droplets and the rate of travel from country to country, the infection has hit the world unlike any virus in recent history. The United States was neither informed nor prepared, and the lack of PPE threatened the medical community as well as the public at large [3]. As New York became the epicenter of this virus, nurses were overworked, lacked proper protection, and became infected, despondent and depressed. The political decision makers have not paid enough attention to the medical experts; and the needs of the states to provide proper PPE supplies, testing and ventilator equipment have not been satisfied [3]. Nurses were being asked to reuse PPE after proper disinfection, but this is not sufficient to protect their own health. Nursing and doctor shortages are causing medical and nursing students to be drafted into practice before 
they complete their standard training. This dangerous precedent was necessary for the current situation but could lead to medical mistakes. However, nurses became extremely vocal and demanding about their own health and safety, and these voices will cause changes in the future in nursing practice. Because of the numbers of new infections, empty convention centers and emergency ships were turned into hospitals, but there was still a shortage of medical staff to work there [4]. Retired nurses and doctors were being asked to return to duty during this crisis.

The N95 respirator is the standard protection for procedures which involve small particles, and surgical masks are used to prevent splash and large droplet, an alternative form of protection when there is an N95 shortage [2,5]. Re-using N95 masks was recommended during the 2009 pandemic and is the subject of National Institute for Occupational Safety and Health current guidelines; these include swearing a simple mask over N95 to prevent contamination and using clean linens in place of simple masks for symptoms patients [6]. Crisis Standard of Care (CSC) includes fairness, duty of care, duty of steward resources, transparency, consistency, proportionality, and accountability [6]. CSC must be applied across all levels of healthcare system (virtual, outpatient and inpatient) and have processes in place to manage resources. Federal, state and local health departments must coordinate resources and share current data to avoid confusion and protect the medical community dealing with this virus. The top priority is to protect nurses and doctors from this virus. Without them, there is no proper plan and the medical community, as well as the population at large, is at risk. Communication among political leaders to protect the medical community is of utmost importance. Without the lifesaving PPE, our medical staffs are at risk. Without an informed, well trained medical staff, there is no future for any of us.

\section{Nursing Education}

I am currently working as a nursing educator. Due to the COVID-19 pandemic, I have been forced to teach my students through the internet. While not the best method, the administration cannot risk infecting my students by having them in hospital situations. Simulation is an alternative method to replace clinical hours for student nurses; it can be controlled by technology to present the symptom of patients' condition, and to improve both their clinical judgment skills and their practical skills based on different kinds of simulation. This is totally doable to help students with their clinical skills so they can practice in the laboratory or virtual simulation. Simulation is used to ensure patient safety, to support innovative teaching strategies and to overcome faculty and preceptor shortage and the lack of clinical sites [7]. In general, simulation must match education goals for students to achieve the level of expertise required of a trained nurse. This is another way to train future nurses to function during an epidemic, even if it is not the best method for training.
Do you think we will learn anything from how nurses were affected by SARS in the early 2000s during this pandemic, or are we doomed to repeat the same mistakes?

In the 2003 SARS outbreak, 774 people died in the United States and SARS did not spread widely in the community [2]. SARS was spread by droplets and by close contact person to person, but it did not spread widely. The COVID-19 virus is also spread by droplets but has spread quickly and efficiently before the medical community realized its dangers. Now that our knowledge of this virus is more advanced, social distancing is being reinforced to prevent transmission. Social distancing is believed to flatten the transmission curve and slow the spread of COVID-19. However, lack of PPE is the new danger to healthcare workers. The important component of WHO intervention was to ensure all healthcare workers wear appropriate PPE [8]. In Hong Kong, nursing schools revised their curriculum, provided continuing education for all the healthcare workers regarding infection control measures, and revised public health policy to ensure the community is protected [9]. Infection control, isolation, and quarantine were implemented. Also, early detection, contamination and preventing spread of the disease were helpful to isolate the cases.

However, some infected people without symptoms can spread the diseases. This is much worse than SARS. We learned some lessons from SARS outbreak. But COVID-19 arrived when the President of United States was more interested in maintaining a robust economy than in providing life-saving healthcare. The result was that the United States had the highest death rate of COVID-19 in the world. The worst fear is that there may be an even more virulent, deadly virus waiting to emerge. Without proper protocols, changes in policy, stockpiling of necessary PPE and ventilators, and a complete reliance on the facts of the scientific community, the United States may not survive the next pandemic. Previous federal administrations had created a medical task force program for a possible pandemic, but the last administration let those task forces lapse, spread disinformation about the pandemic and created the current crisis by ignoring science. We need to have an annual budget commitment that can survive changes in federal administration with leadership experience to provide funding, staffs and awareness of possible future pandemics $[10,11]$.

\section{In conclusion}

Since these deadly diseases can be spread quickly and easily around the world, a unified world-wide plan should be discussed and implemented among the most important international institutions that connect all countries, like the United Nations and the World Health Organization. At the top level, government officials must be honest about any future outbreak, work efficiently to stop its transmission, and provide maximum protection of the medical community that is needed to save the world. The medical community must determine all forms of prevention, management, 
protection and treatment, and it should not be disparaged by politics or disinformation. If the nursing community is not adequately trained, properly protected and physically and psychologically fit, there will be no one left to heal the sick.

\section{Acknowledgement}

None.

\section{Conflict of Interest}

Author declare no conflict of interest.

\section{References}

1. Chen E, Chung J, Yang J, Wong T, Lai A, et al. (2008) Evaluating nursing practice models in the context of SARS. Hong Kong Medical Journal 14(1): $42-45$

2. Center for Disease Control and Prevention (2020) Covid 19.

3. Cohen J, Rodgers Y (2020) Contributing factors to personal protective equipment shortages during the COVID-19 pandemic. Prev Med 141: 106263.
4. American Colleges Association of Nursing (2020) Nursing shortage.

5. David E (2020) The battle to protect healthcare workers on the front lines of the coronavirus pandemic.

6. Hick JL, Hanfling D, Wynia MK, Pavia AT (2020) Duty to plan: healthcare, crisis standard of care and Novel-coronavirus-SARS-coV-2.

7. Li S (2020) The role of simulation in nursing education: a regulatory perspective.

8. Murphy C (2006) The 2003 SARS outbreak: global challenges and innovative infection control measures. Online J Issues Nurs 11(1): 6.

9. Thompson DR, Lopez V, Lee D, Twinn S (2004) SARS--a perspective from a school of Nursing in Hong Kong. J Clin Nurs 13(2): 131-135.

10. Mosk M (2020) George W Bush in 2005: if we wait for a pandemic to appear, it will be too Late to prepare.

11. Center for Disease Control and Prevention (2017) Severe Acute Respiratory Syndrome. 